\title{
Parasagital us approach for L5 medial branch radiofrequency ablation
}

Patricia Alfaro de la Torre, Rocio Periñan Blanco, Jordi Recasens Urbez, Sergi Boada Pie. Chronic Pain Unit, Hospital Joan XXIII, Tarragona, Spain

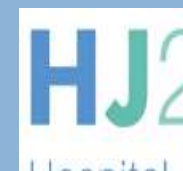

@sebopi @patrialfarot @jordireca @rociopebl

\section{Background and aim:}

- Block and ablation of Medial Branch of Dorsal Nerve is the most accepted technique for diagnosis ant treatment of lumbar facet pain.

- Medial Branch Ultrasound Guided classic technique use a transversal window not suitable for L5 most times because of the acoustic shadow of iliac crest.

\section{Through this study we propose an ultrasound} parasagittal approach versus classic transverse approach to L5 Dorsal Rami useful for the realization of both: the diagnostic block and the L5 dorsal rami thermal ablation. We ASSESS the accuracy of this new approach with fluoroscopy check

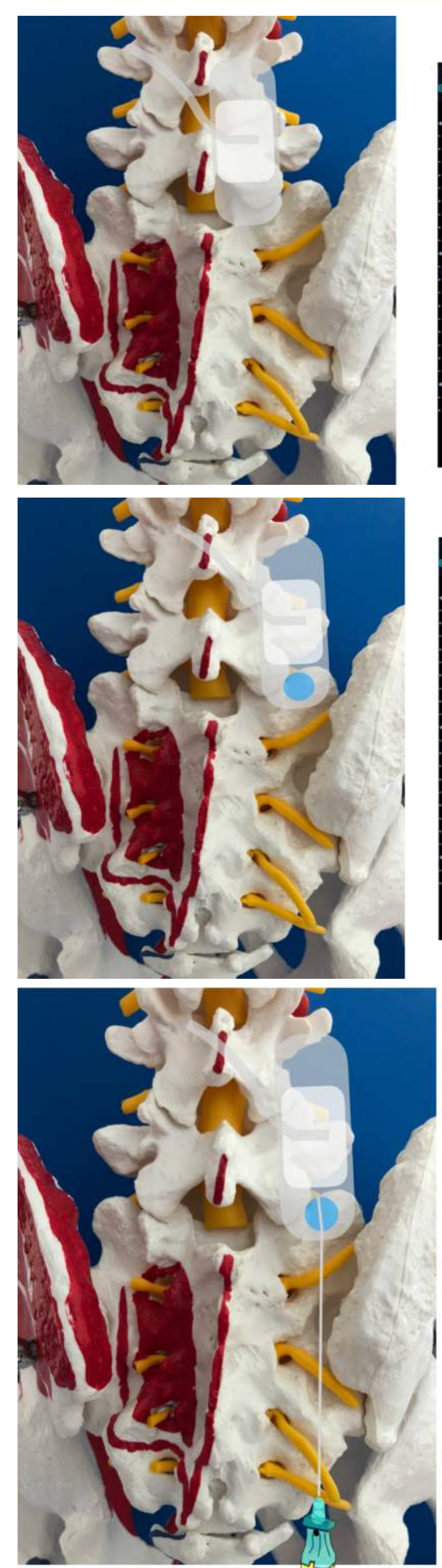

Conclusions:
PARASAGITTAL ARTICULAR VIEW

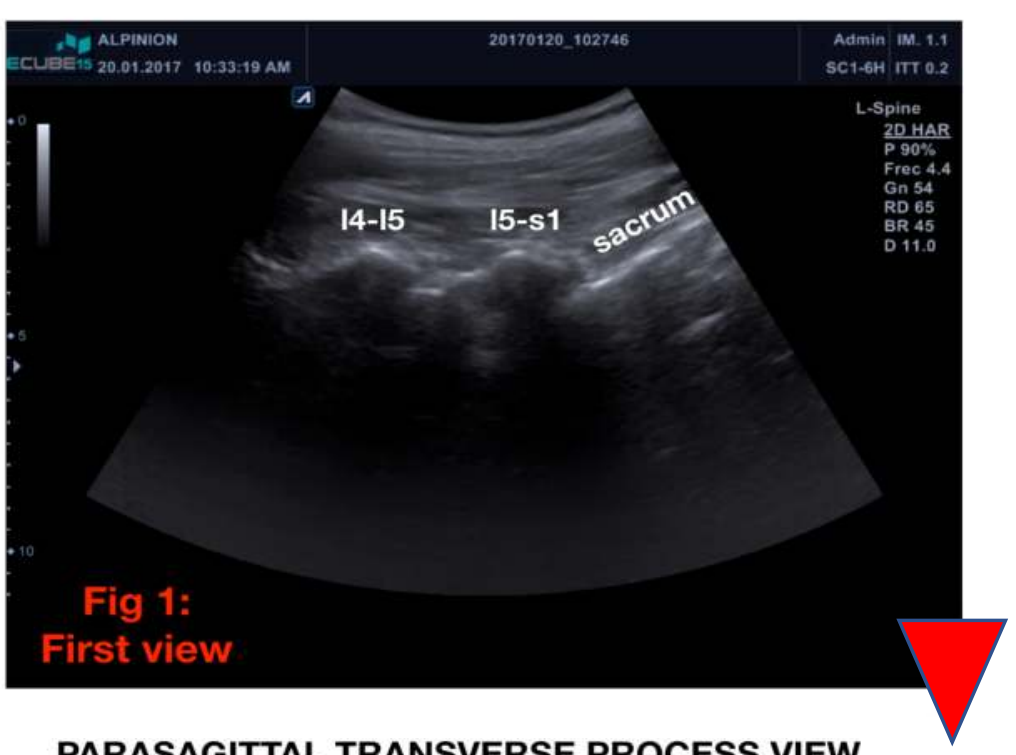

PARASAGITTAL TRANSVERSE PROCESS VIEW
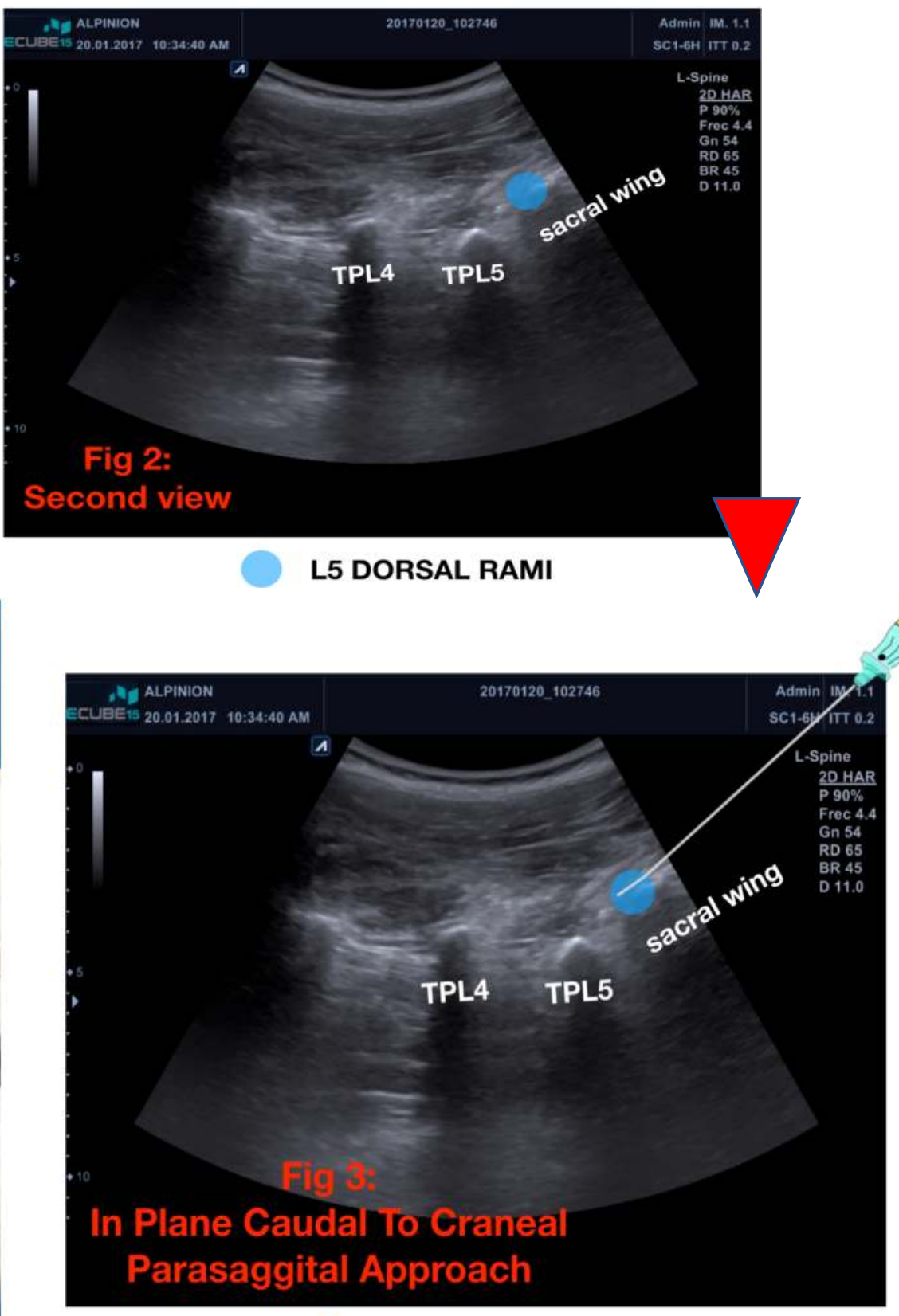

L5 DORSAL RAMI
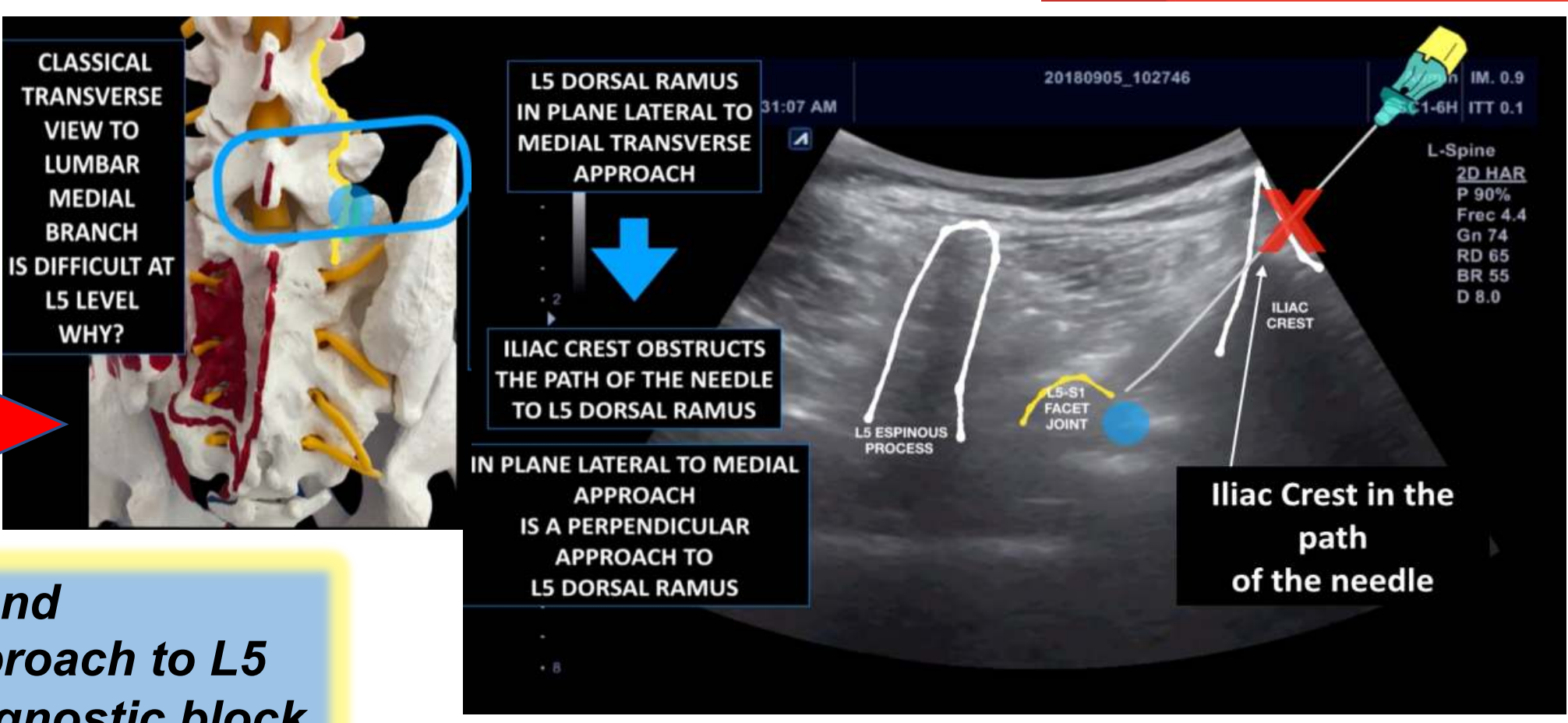

Material and methods:

After obtaining the informed consent, we included 41 patients with lumbar facet pain.

Prone position, sedation with $5 \mathrm{mg}$ of iv midazolam; the same observer performed the technique with a Sonosite $® E d g e ~ T M ~ 5-2$ $\mathrm{MHz}$ curve probe and 20G Neurotherm SL-C1010-20® RF curved tip needle.

The parasagittal articular line and acoustic shadow of the sacrum are identified (fig.1), the probe slides laterally until the joint line is lost and the base of the superior articular process of $\mathrm{S} 1$ is visualized in the sacral wing, together with the transverse processes of L3, L4 and L5 (fig.2).

Introduction of the needle in plane caudal to craneal until reaching the superior and posterior aspect of the sacral wing, lateral to the articular process of S1. Fine repositioning of the tip of the needle was made until correct motor and sensory stimulation was elicited(fig.3).

$\rightarrow$ Root motor stimulus discarded and positive sensory stimulus + Rx AP to check the correct placement of the tip of the needle.

\section{Results:}

The technique was successfully performed in 35 patients $(\mathbf{8 5 . 3 6 \%})$ In 6 patients the necessary windows could not be obtained. The tip of the needle was positioned at the first attempt or with a single replacement in 33 of the 35 patients, so the total success rate was $94.28 \%$ ONLY IN TWO PATIENTS NEEDLE TIP WAS NOT POSSIBLE TO BE PLACED ON THE TARGET EVEN AFTER FINE REPOSITIONING.

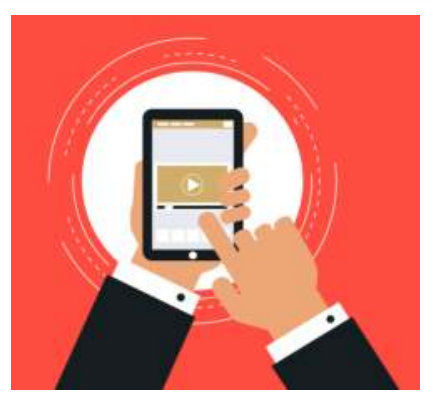

Do you want to Learn the technique through a video?

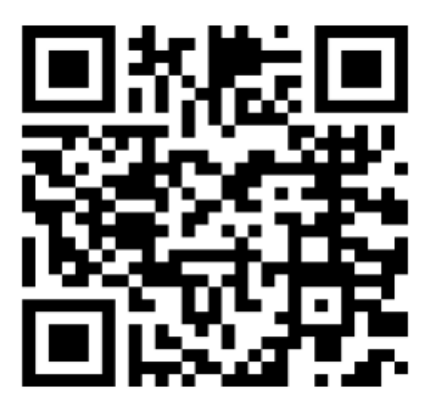

More studies are required to evaluate efficacy and safety and it requires a learning curve (operator-dependent).

The parasagital approach of the L5 branch requires the identification of fewer echographic windows than the classical one.

Adventajes: allows a better placement of the needle tip with respect to the nerve for thermal ablation, avoid iliac crest and allows in-plane approach.

The possible application of this technique in a standardlzed way in our pain units could provide countless positive results such as the reduction of the use of fluoroscopy, reduction of the waiting list, increase in patient satisfaction, optimization of resources, lower cost, etc. 